\title{
TRAUMA Y VIOLENCIA EN PATAGONIA AUSTRAL. INTERPRETACIÓN DE EVIDENCIAS BIOARQUEOLÓGICAS Y PERSPECTIVAS FUTURAS
}

\author{
TRAUMA AND INTERPERSONAL VIOLENCE IN SOUTHERN PATAGONIA. \\ INTERPRETATION OF BIOARCHAEOLOGICAL EVIDENCE AND FUTURE \\ PERSPECTIVES
}

\author{
Gustavo Flensborg ${ }^{1}$ y Jorge A. Suby ${ }^{2}$
}

\begin{abstract}
En este trabajo se revisan y analizan los reportes de traumas en restos humanos de Patagonia Austral, discutiendo sus posibles implicancias bioarqueológicas y exponiendo los futuros pasos que a nuestro criterio deberían seguir las investigaciones de este tipo de lesiones patológicas en la región. Sobre un total de 126 esqueletos humanos, se reportaron 15 (11,9\%) individuos adultos con traumas, principalmente individuos masculinos. Todas las lesiones fueron identificadas en restos del Holoceno Tardío, aunque algunas se registraron en esqueletos sin información cronológica. Se observaron depresiones en los cráneos, fracturas en huesos largos, cortos y vértebras e inclusiones de puntas de proyectil en coxales y cráneos. La mayoría de las lesiones son antemortem y fueron relacionadas con accidentes y violencia, seguidas por perimortem, que en todos los casos fueron vinculadas a eventos de violencia interpersonal. Aunque sin diferencias significativas, las subregiones de Santa Cruz/Magallanes y norte de Tierra del Fuego presentan frecuencias más elevadas de traumas y de casos relacionados con violencia interpersonal que el sur de Tierra del Fuego, indicando posibles variaciones en la exposición a accidentes y en las relaciones sociales de conflicto, así como contrastes en la intensidad de las investigaciones. Los porcentajes y los tipos de lesiones son similares a aquellos traumas informados para otras regiones de Patagonia. Por último, no se observan evidencias de aumento de tensión social que acompañen el proceso de aumento demográfico identificado durante el Holoceno Tardío en Patagonia Austral.
\end{abstract}

Palabras claves: lesiones traumáticas, restos óseos humanos, Patagonia Austral, Holoceno Tardío.

In this work, reports of traumas in human remains from Southern Patagonia are reviewed and analyzed. Also, bioarchaeological implications and the future steps of trauma investigations are discussed. Considering a total sample of 126 skeletons, fifteen (11.9\%) adult individuals, mainly male individuals. were identified with reports of traumas. All lesions were recorded in individuals of the late Holocene, although some were recorded in remains that lacked chronological information. Depressions in the skulls, fractures in long and short bones as well as vertebrae, and inclusions of projectile points in coxal bones and skulls were observed. Most lesions are antemortem, associated with accidents and violence, followed in frequency by perimortem lesions that were always linked to interpersonal violence events. The subregions of Santa Cruz / Magallanes and North of Tierra del Fuego have the highest frequencies of trauma and cases related to interpersonal violence, in comparison with the South of Tierra del Fuego, but without statistically significant differences. This could indicate variations in exposure to accidents and social conflicts as well as differences in the intensity of the investigations. The percentages and types of injuries are similar to those traumas reported for other regions from Southern Patagonia. Finally, there is no evidence of increased social tension that accompanies the process of demographic increase identified during the late Holocene in Southern Patagonia.

Key words: Traumatic lesion, human bone, southern Patagonia, Late Holocene.

Los estudios de la frecuencia, tipos y distribución anatómica de los traumas en esqueletos humanos del pasado aportan información acerca de la adaptación de las sociedades humanas a los cambios demográficos, las transiciones climáticas y/o ambientales, las variaciones en las relaciones sociales, los tratamientos rituales, los conflictos inter e intra grupal y los patrones de actividad física asociados a labores diarias (Allen 2014; Gordón 2015; Lessa 2011; Lovell 2008; Martin y Osterholtz 2016; Santana et al. 2012). El enfoque biocultural adoptado en este tipo de estudios permite no sólo discutir eventos traumáticos que fueron significativos en la vida y en la salud de los individuos, sino también lograr una interpretación

\footnotetext{
${ }^{1}$ INCUAPA-CONICET- Departamento de Arqueología, Facultad de Ciencias Sociales, Universidad Nacional del Centro de la Provincia de Buenos Aires, Olavarría (7400), Buenos Aires, Argentina. Grupo de Investigación en Bioarqueología. gflensbo@ @oc.unicen.edu.ar ${ }^{2}$ INCUAPA-CONICET- Departamento de Arqueología, Facultad de Ciencias Sociales, Universidad Nacional del Centro de la Provincia de Buenos Aires, Quequén, Buenos Aires, Argentina. Grupo de Investigación en Bioarqueología. jasuby @ conicet.gov.ar
}

Recibido: septiembre 2018. Aceptado: mayo 2019.

http://dx.doi.org/10.4067/S0717-73562020005000101. Publicado en línea: 7-enero-2020. 
desde perspectivas paleoepidemiológicas (Bennike 2008; Jurmain 1991; Jurmain et al. 2009).

A diferencia de los estudios realizados en otras regiones de Argentina, como el Noroeste Argentino (Nielsen 2007; Seldes y Bota 2014), Pampa (Berón 2012), transición pampeano-patagónica oriental (Flensborg 2011, 2012) y norte de Patagonia (Barrientos y Gordón 2004; Gordón 2011, 2014, 2015), donde los estudios de trauma y violencia en restos óseos humanos han sido analizados y discutidos con mayor profundidad, los reportes de este tipo de evidencias en Patagonia Austral son escasos y no han sido objeto de evaluaciones específicas (Prieto y Cárdenas 2007). Por el contrario, la mayor parte de las lesiones atribuidas a eventos traumáticos provienen de estudios de caso o intrasitio (p.ej., L'Heureux y Amorosi 2009; Suby et al. 2008), por lo que no contamos hasta el momento con análisis producidos a partir de conjuntos esqueletales de mayor tamaño en escalas regionales o macro-regionales.

Patagonia Austral (Figura 1) es un territorio extenso, ubicado al sur de los $50^{\circ}$ de latitud sur, caracterizado por una amplia variabilidad ecológica y ambiental. Las evidencias de ocupación humana en el sur del continente y en el norte de Tierra del Fuego datan de ca. 11.500 años AP (Miotti et al. 2003; Prieto 1991) y de ca. 7.800 años AP en el sur de Tierra del Fuego (Orquera y Piana 2009). Las poblaciones humanas han sido descritas como grupos de baja densidad demográfica y alta movilidad, aunque hacia los ca. 3.500 años AP y hasta el inicio del periodo de contacto hispano-indígena se habría producido un incremento demográfico (Barberena 2008; Pallo y Ozán 2014; Suby et al. 2017). La información etnográfica y etnohistórica indica que la actual provincia de Santa Cruz y norte de Tierra del Fuego fueron espacios ocupados por cazadores-recolectores terrestres. En cambio, en el sur de Tierra del Fuego habitaron grupos cazadoresrecolectores marítimos (Gusinde 1986 [1937]; Orquera y Piana 2009; Prieto y Cardenas 2007; Saletta 2015). Sin embargo, las evidencias arqueológicas muestran un continuo entre estos dos tipos de economía (Barberena 2008; Borrero y Barberena 2006; Morello et al. 2012).

Esta variabilidad conductual, ecológica y demográfica, descrita en escalas espacio-temporales amplias, pudo haber generado escenarios diversos para que ocurrieran eventos traumáticos a nivel físico. En el presente trabajo se revisan y analizan los antecedentes reportados de individuos en los cuales se registraron lesiones compatibles con traumas en Patagonia Austral, discutiendo sus posibles implicancias bioarqueológicas y exponiendo los futuros pasos que, a nuestro criterio, deberían seguir las investigaciones relacionadas con este tipo de lesiones patológicas en la región. El análisis de los casos de traumas en Patagonia Austral es además comparado con los resultados obtenidos de otras regiones de Patagonia.

\section{Interpretación de los Traumas en Contextos Bioarqueológicos}

El trauma se define como una lesión del tejido vivo que es causado por una fuerza o mecanismo extrínseco al cuerpo (Lovell 1997). Los principios teóricos y metodológicos utilizados en bioarqueología se sustentan en el campo de la medicina clínica y la antropología forense, debido a que estas disciplinas científicas tienen las herramientas y los conocimientos necesarios para interpretar la evidencia y determinar la causa de muerte a través del registro de lesiones presentes en el hueso, el análisis del intervalo en el que ocurrió el trauma y los mecanismos involucrados en el evento traumático (Galloway 1999). Estos principios son en general tomados y adaptados para su aplicación en restos humanos de poblaciones pasadas, a los fines de evaluar relaciones entre los traumas y aspectos sociales, como las actividades cotidianas o las relaciones inter $\mathrm{e}$ intragrupo en escalas poblacionales (Martin y Anderson 2014). Junto con el análisis patológico, la interpretación de la información del contexto social y cultural es el medio por el cual se logra explicar el significado de los traumas (Judd y Redfern 2012). Solo de esta manera es posible caracterizar modos y estilos de vida y evaluar los comportamientos humanos relacionados con la violencia (Berón 2012; Gordón 2011; Jordana et al. 2009; Jurmain et al. 2009; Lessa 2011; Pfeiffer 2016; Scott y Buckley 2010; entre otros).

En paleopatología, el trauma óseo incluye una variedad de categorías tales como fracturas, lesiones por aplastamiento, heridas por fuerza cortante, dislocaciones, deformación, scalping, mutilación, trepanación, espondilolisis, entre muchos otros (Aufderheide y Rodríguez-Martin 1998; Bennike 2008; Judd y Redfern 2012; Mays 2010; Merbs 1989). Las patologías consideradas como traumas pueden variar entre autores (ver por ejemplo Aufderheide y Rodríguez Martín 1998; Ortner 2003). Un ejemplo de esto son las lesiones por violencia, a las cuales no todos los autores incluyen como traumas. Sin embargo, las dificultades en algunos casos para la determinación de episodios de violencia hace imposible establecer un límite claro, y por lo tanto son englobados dentro del análisis de lesiones traumáticas. En este trabajo se adopta esta última postura.

Una de las partes esenciales del análisis de los traumas consiste en determinar el momento en que se produjo la modificación ósea. Esto se divide en tres categorías: (1) antemortem, o antes de la muerte, (2) perimortem, o próximo al momento de la muerte y (3) postmortem, o después de la muerte, ya sean intencionales o producidas por procesos tafonómicos (Berryman y Symes 1998; Sauer 1998). Las lesiones antemortem se reconocen cuando hay evidencia de remodelación ósea que indica la curación total o parcial 


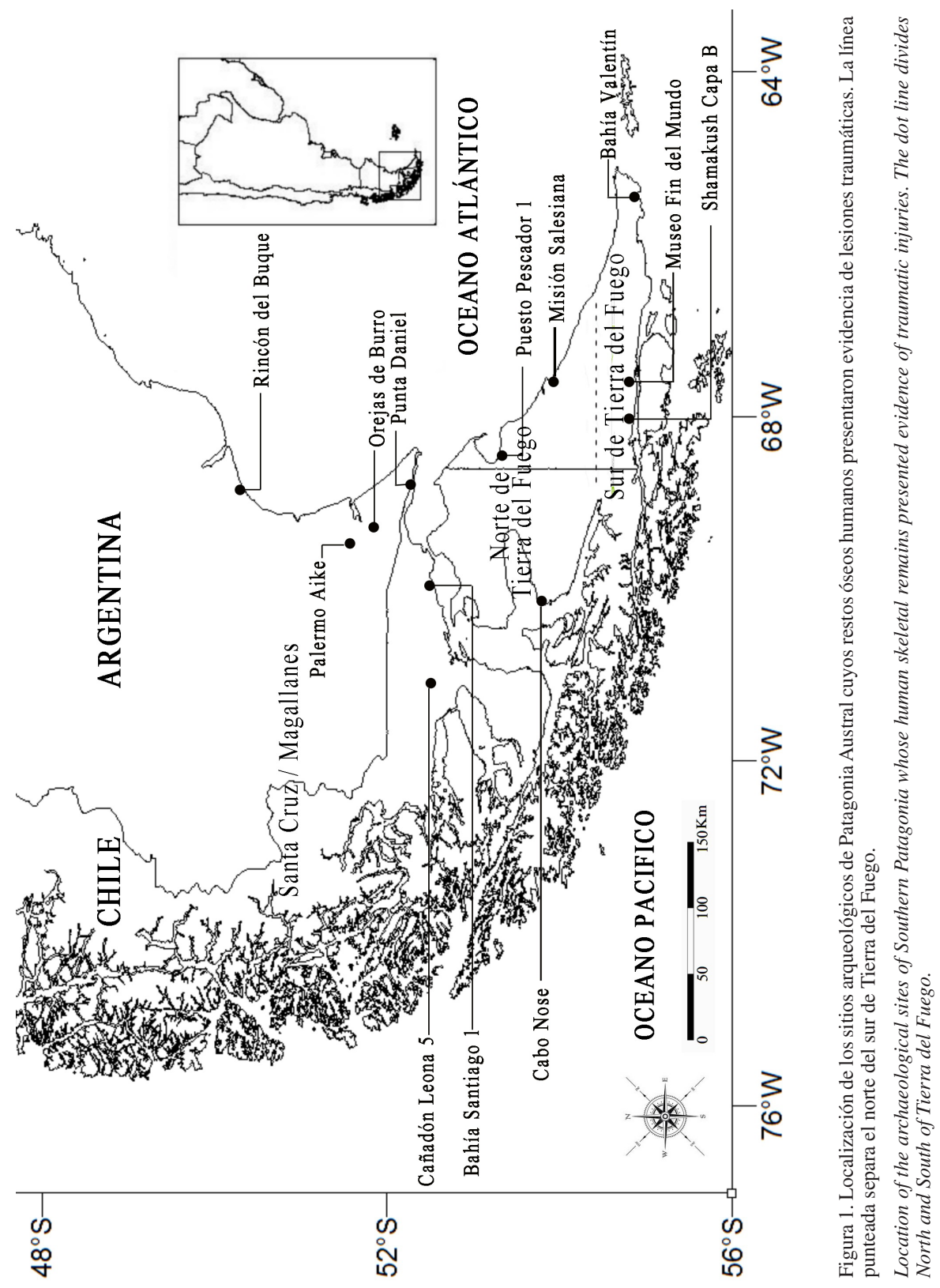


de la herida. Este proceso depende de factores como la edad, el sexo y la salud del individuo, el tipo de trauma, el hueso afectado, el objeto involucrado, la energía de impacto sobre el cuerpo, entre otros (Bennike 2008; Galloway 1999; Wedel y Galloway 2014).

Las fracturas y los daños postmortem pueden ser causados por una diversidad de agentes extrínsecos, como los daños provocados por la dentición de carnívoros y roedores, la acción térmica, el impacto de rocas, la presión sedimentaria, la actividad antrópica, etc. (Galloway 1999; Stodder 2008). Un punto crítico, en ocasiones, es lograr discriminar entre lesiones perimortem y postmortem, ya que ambas carecen de evidencias de remodelación ósea. Para diferenciar entre ambas categorías a menudo se evalúa la coloración de la superficie de las fracturas, la plasticidad del hueso y ciertas propiedades como el ángulo, contorno, angulación, dirección y morfología de la lesión (Villa y Mahieu 1991). Por este motivo, es recomendable el estudio de los restos óseos durante el trabajo de campo $\mathrm{y}$ antes de su limpieza para poder realizar inferencias confiables, ya que en ocasiones es posible reconocer evidencias directas relacionadas con el trauma (p.ej., proyectil asociado a los huesos) y/o modificaciones postdepositacionales que pueden sesgar y comprometer la posibilidad de identificar superficies de fractura.

La mayoría de los eventos traumáticos pueden ser agrupados en tres categorías (Adams 2007): (1) contundente, que genera los tipos de fracturas más frecuentes, entre ellas las de tipo espiral, mariposa, transversal y conminuta en los huesos largos, y las depresiones en el cráneo; (2) aguda, generadas por armas blancas que producen lesiones más profundas que anchas, con borde estrecho y apariencia de "V"; y (3) disparo (proyectil), incluye a todas las heridas generadas por la acción de proyectiles (armas de fuego y puntas de proyectil de diversos materiales, p.ej., metal, madera, hueso, material lítico) (Adams 2007). Asimismo, es necesario el estudio y la comprensión de las características de las lesiones y los contornos del punto de impacto para realizar interpretaciones acerca de los instrumentos u objetos que generaron el trauma, de la secuencia en la que ocurrieron las lesiones y de las fuerzas primarias involucradas en el trauma (p.ej., tensión, compresión, cizallamiento, rotación), en función del tipo de hueso, sus propiedades biomecánicas y la magnitud del daño (Galloway 1999; Gurdjian 1975; Lovell 1997).

\section{Materiales y Métodos}

Se realizó una búsqueda bibliográfica de trabajos en los que se reportaron casos de traumas óseos en restos humanos arqueológicos procedentes de Patagonia Austral (Figura 1). Solo fueron considerados reportes asociados específicamente a fracturas, depresiones y heridas producidas por proyectiles producto de hechos de violencia. Por el contrario, no se incluyeron traumas dentales, trepanaciones o espondilolisis, los cuales pueden estar vinculados a diferentes procesos patológicos. Se recabó la información publicada acerca de las características de los traumas, dimensiones, elementos afectados, sexo y edad de los individuos y el contexto del cual provienen los restos (subregión y cronología). A partir de estos datos se evaluó la variabilidad espacial y temporal de las lesiones asociadas a traumas. La información sexo-etaria, aunque publicada por diferentes autores, en general fue analizada siguiendo los mismos métodos, basados en el standard de Buikstra y Ubelaker (1994), como fue detallado en Suby et al. (2017).

Se estimó la frecuencia de individuos afectados por traumas en una muestra de 126 esqueletos con un rango temporal que comprende el Holoceno medio y tardío (ca. 5.200-300 años AP), dentro de la cual están contenidos todos los individuos con reportes de traumas identificados hasta el momento. Esta muestra está compuesta por 119 individuos detallados en Suby et al. (2017; Tabla 1), que fueron seleccionados por disponer información de sexo, edad, ubicación geográfica, y en algunos casos, cronología e información paleodietaria, a la que se añadieron los individuos procedentes de los sitios Palermo Aike (n=1; Suby 2014); Bahía Santiago ( $\mathrm{n}=1$; Constantinescu 2003); Individuo 2405 resguardado en el Museo del Fin del Mundo ( $n=1$; Underdown 2012); restos humanos mezclados en la colección de la Antigua Misión Salesiana de Rio Grande ( $\mathrm{n}=2$; Suby y Guichón 2010); Bahía Valentín $(\mathrm{n}=1$; Kozameh et al. 2000) y Shamakush Entierro Capa B ( $\mathrm{n}=1$; Suby et al. 2011). Además se calcularon las frecuencias de traumas sobre el total de individuos afectados y sobre el total de lesiones registradas. También se estimó la frecuencia en función del sexo, la edad y la subregión de origen. En este último caso las unidades espaciales se denominaron como Santa Cruz/ Magallanes, norte de Tierra del Fuego y sur de Tierra del Fuego. Se considera arbitrariamente el límite entre sur y norte de Tierra del Fuego como la línea imaginaria al nivel del Lago Fagnano (Tierra del Fuego) (Figura 1).

Se utilizó la categoría subadulto (entre 0 y 20 años), adulto joven (entre 20-34,9 años) y adulto medio (entre 35-50 años) (Buikstra y Ubelaker 1994). No se tuvieron en cuenta a los adultos maduros, debido a que solo un esqueleto del total de 126 individuos corresponde a este grupo de edad. Cuando se presentó información interpretativa de los traumas, estos fueron clasificados en dos categorías: accidental o violencia interpersonal. Las diferencias en las frecuencias entre sexo, edad y subregiones fueron evaluadas con el test exacto de Fisher, con un nivel de significancia de $p \leq 0,05$. 
Tabla 1. Información sexo-etaria de los esqueletos considerados para este trabajo por región. Nota: $\mathrm{TDF}=$ Tierra del Fuego; $\mathrm{M}=$ masculino, F= femenino; Indet.= indeterminado; Ad. Jov.= adulto joven; Ad. Med.= adulto medio.

Sex-age data of the skeletons considered for this work by region. Note: $T D F=$ Tierra del Fuego; $M=$ male $; F=$ female $;$ Indet.$=$ indeterminate $;$ Ad. Jov.= young adult $;$ Ad. Med.$=$ middle adult .

\begin{tabular}{ccccccccc}
\hline Región & N & M & F & Indet. & $\begin{array}{c}\text { Sub- } \\
\text { adulto }\end{array}$ & $\begin{array}{c}\text { Ad. } \\
\text { Jov. }\end{array}$ & $\begin{array}{c}\text { Ad. } \\
\text { Med. }\end{array}$ & $\begin{array}{c}\text { Adulto } \\
\text { Indet. }\end{array}$ \\
\hline Santa Cruz/ & & & & & & & & \\
Magallanes & 41 & 15 & 9 & 17 & 20 & 10 & 10 & 1 \\
Norte de TDF & 33 & 16 & 10 & 7 & 10 & 16 & 5 & 2 \\
Sur de TDF & 52 & 17 & 21 & 14 & 13 & 20 & 17 & 2 \\
TOTAL & 126 & 48 & 40 & 38 & 43 & 46 & 32 & 5 \\
\hline
\end{tabular}

A partir de las descripciones realizadas por los autores de las publicaciones respecto de las lesiones traumáticas, se tuvo en cuenta la extensión de la lesión en cada hueso y sus características morfológicas principales, el momento en que se produjeron y los posibles objetos involucrados en el origen de los mismos. Estos datos son tomados de las fuentes originales y no se incluyen aquí nuevas evaluaciones, las cuales formarán parte de instancias posteriores y más avanzadas de nuestras investigaciones sobre traumas en la región.

\section{Resultados}

Se identificó una prevalencia de 11,9\% (15/126) de individuos con traumas, entre los cuales se registraron 28 lesiones (Tabla 2). La mayoría de los individuos presenta un solo trauma, excepto por cinco esqueletos masculinos que tienen evidencias de más de una lesión (Tabla 2). De ese total, el 22,9\% (11/48) de los individuos masculinos presentaron algún tipo de trauma, mientras que éstos solo afectaron al 2,5\% (1/40) de los individuos femeninos. Asimismo, el 7,9\% (3/38) de los individuos indeterminados presentaron lesiones (Tabla 3). La diferencia entre los sexos es estadísticamente significativa $(\mathrm{p}=0,02)$. Si se tiene en cuenta la cantidad de individuos afectados, el sexo masculino presenta la mayor frecuencia $(73,3 \% ; 11 / 15)$, en relación al femenino $(6,6 \% ; 1 / 15)(\mathrm{p}=<0,001)$. Por su parte, en función de la cantidad de lesiones relevadas, también se aprecia una supremacía de traumas en individuos masculinos $(85,7 \% ; 24 / 28)$ en comparación con los femeninos $(3,6 \% ; 1 / 28)(\mathrm{p}=<0,001)$, mientras que tres esqueletos que presentaban lesiones resultaron ser individuos de sexo indeterminado.

Todos los traumas se registraron en individuos adultos, en mayor frecuencia en adultos medios $(21,9 \%$; $7 / 32)$ en comparación con adultos jóvenes $(6,5 \%$; $3 / 46$ ), aunque no existen diferencias estadísticamente significativas $(\mathrm{p}=0,136)$. Esta tendencia también se observó cuando se considera a los individuos afectados, ya que los adultos medios presentan mayor porcentaje de lesiones $(46,6 \% ; 7 / 15)$ en comparación con los adultos jóvenes $(20 \% ; 3 / 15)$, aunque sin diferencia estadística $(\mathrm{p}=0,27)$. Respecto del total de lesiones, los adultos medios también presentan la mayor frecuencia $(32,1 \% ; 9 / 28)$, en relación con adultos jóvenes $(17,8 \% ; 5 / 28)(\mathrm{p}=0,466)$.

Las lesiones antemortem se registraron en mayor frecuencia $(n=21 ; 75 \%)$ en comparación con las perimortem $(\mathrm{n}=6 ; 21,4 \%)(\mathrm{p}=0,01)$, mientras que en un solo caso no se especificó esta información. Los traumas fueron registrados principalmente en cráneos $(n=7)$ y costillas $(n=6)$, seguido por el coxal, húmero, radio y cúbito ( $\mathrm{n}=2$ en cada elemento) y por la mandíbula, atlas, vértebra dorsal y lumbar, tibia y $5^{\circ}$ metacarpo $(n=1$ en cada elemento). En tres individuos se observaron fracturas en la zona del cuerpo y sector distal de las costillas de tipo antemortem con presencia de callos osificados alineados al hueso (Figura 2a), excepto por un caso que no logró la unión de los fragmentos (ver otros ejemplos en L'Heureux y Barberena 2008: Figura 8). En el cráneo de tres individuos se registraron depresiones y fracturas lineales pre y perimortem, de diferentes dimensiones (rango de 15 x $10 \mathrm{~mm}$ ) en varios huesos de la calota (temporales, frontal y parietales), y en dos individuos se observaron impactos de puntas de proyectil lítico en la norma derecha de los parietales (Constantinescu 2003: Figura 2; Suby et al. 2008: Figura 7; L'Heureux y Amorosi 2009: Figuras 6, 9, 10 y 11; Suby y Guichón 2010: Figura 7). En los húmeros, radios y cúbitos se registraron fracturas de tipo antemortem, principalmente localizadas en las zonas proximales (Figura 2b) que ocasionaron en varios casos la reducción de la longitud de los huesos (Underdown 2012: Figura 2). En una vértebra dorsal y en otra lumbar se evidenció aplastamiento anterior del cuerpo (Suby et al. 2008: Figura 5), mientras que en el atlas ocurrió una fractura del arco neural anterior (Figura 2c). En todos los casos las lesiones fueron antemortem. En los coxales se detectaron puntas de proyectil lítico insertas en el ilion que ocasionaron lesiones perimortem (Figura 2d). En la rama ascendente de una mandíbula y en la diáfisis de un metacarpo se registraron fracturas antemortem y finalmente se observó una punta de proyectil asociada al tórax de un individuo (Tabla 2).

Los traumas documentados fueron interpretados por los respectivos autores como parte de eventos de violencia interpersonal para el 53,6\% (15/28) de las lesiones, afectando al 46,6\% (7/15) de los individuos. En todos los casos las lesiones asignadas a hechos violentos se generaron como producto del impacto de proyectiles líticos sobre el cuerpo o por objetos romos en la bóveda del cráneo. Por su parte, el 14,3\% (4/28) de los traumas fue relacionado con eventos accidentales afectando el 


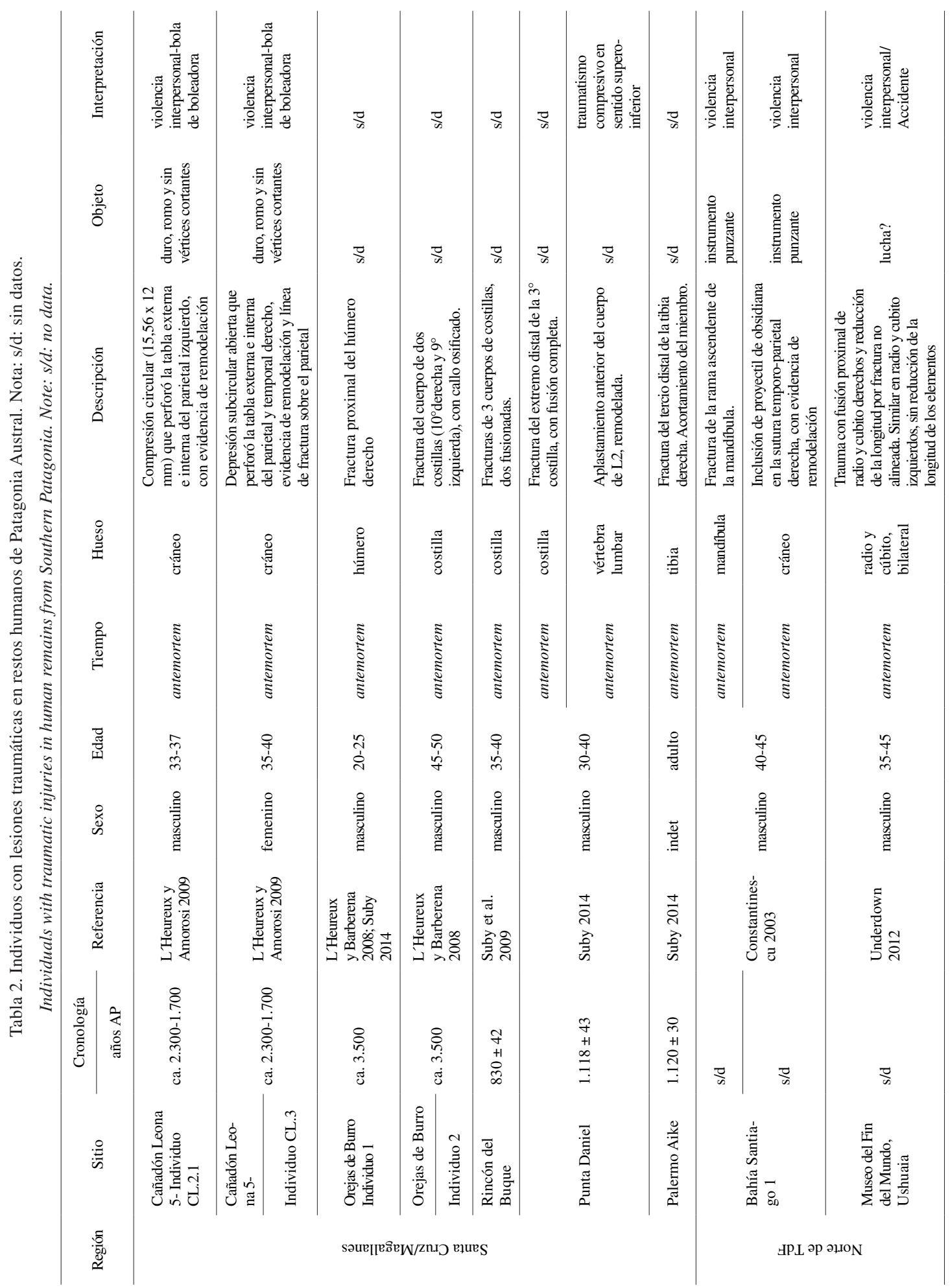




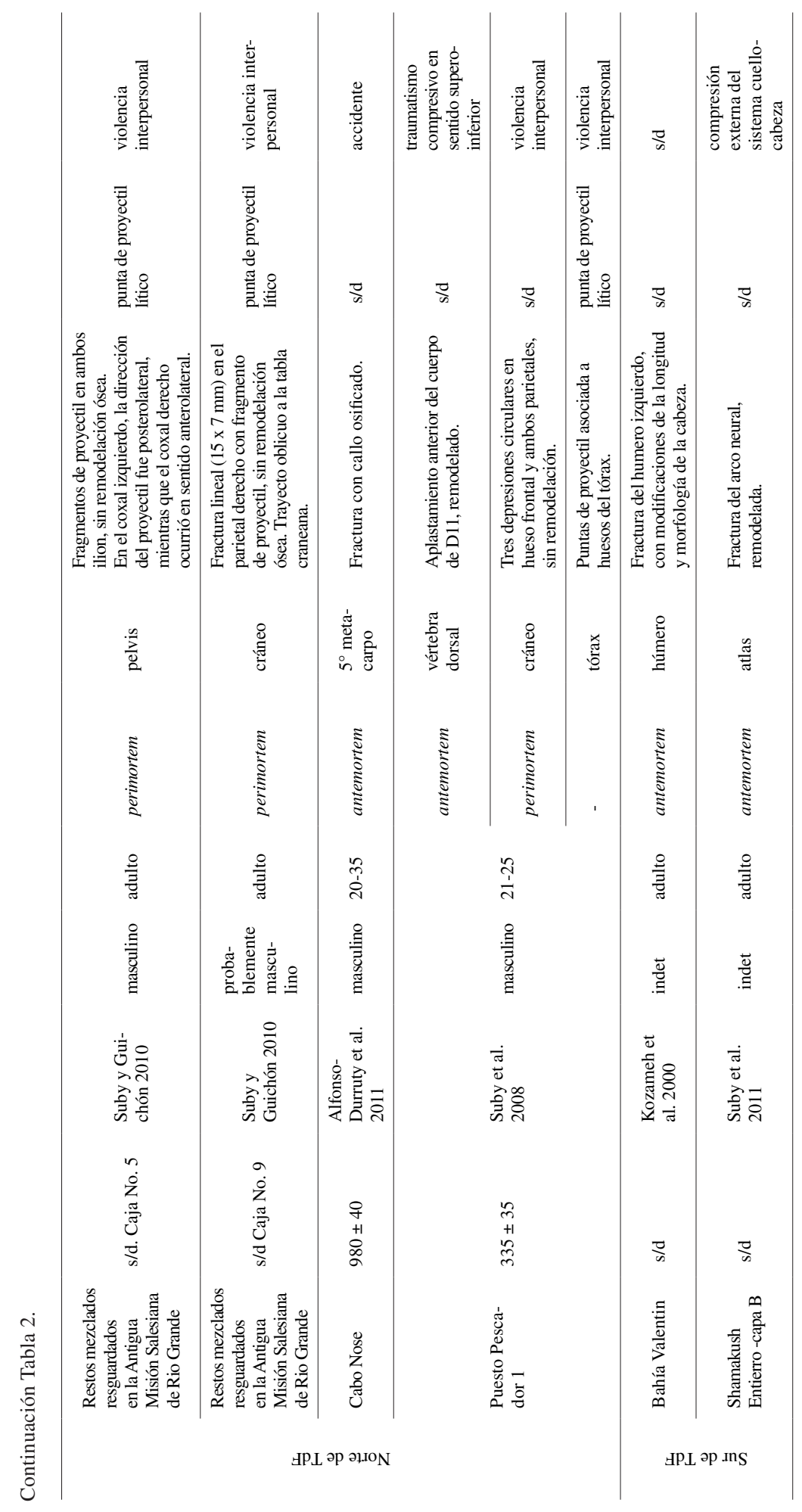


Table 3. Frecuencias totales y por región, sexo y edad de individuos con traumas identificados en restos óseos humanos de Patagonia Austral. Nota: $\mathrm{n}=$ casos afectados; $\mathrm{N}=$ casos analizados; Indet= indeterminado.

Total frequencies and frequencies by region, sex and age of individuals with traumas identified in human skeletal remains of Southern Patagonia. Note: $n=$ affected cases; $N=$ cases analyzed; Indet= indeterminate.

\begin{tabular}{|c|c|c|c|c|c|c|c|c|}
\hline \multirow{2}{*}{ Región } & \multirow{2}{*}{$\mathrm{n} / \mathrm{N} / \%$} & \multicolumn{2}{|c|}{ Tipo de trauma } & \multicolumn{3}{|c|}{ Sexo } & \multicolumn{2}{|c|}{ Edad } \\
\hline & & Trauma no violento & Violencia & M & $\mathrm{F}$ & Indet & Subadulto & Adulto \\
\hline Total & $15 / 126 / 11,9$ & $9 / 15 / 60$ & $6 / 15 / 40$ & $11 / 48 / 22,9$ & $1 / 40 / 2,5$ & $3 / 38 / 7,89$ & $0 / 0 / 0$ & $15 / 15 / 100$ \\
\hline $\begin{array}{l}\text { Santa Cruz/ } \\
\text { Magallanes }\end{array}$ & $7 / 41 / 17,1$ & $5 / 41 / 12,2$ & $2 / 41 / 4,9$ & $5 / 15 / 33,3$ & $1 / 9 / 11,1$ & $1 / 17 / 5,8$ & $0 / 0 / 0$ & $7 / 7 / 100$ \\
\hline Norte TdF & $6 / 33 / 18,2$ & $2 / 33 / 6,1$ & $4 / 33 / 12,1$ & $6 / 16 / 37,5$ & $0 / 10 / 0$ & $0 / 7 / 0$ & $0 / 0 / 0$ & $6 / 6 / 100$ \\
\hline Sur TdF & $2 / 52 / 3,8$ & $2 / 52 / 3,8$ & $0 / 52 / 0$ & $0 / 17 / 0$ & $0 / 21 / 0$ & $2 / 14 / 14,3$ & $0 / 0 / 0$ & $2 / 2 / 100$ \\
\hline
\end{tabular}
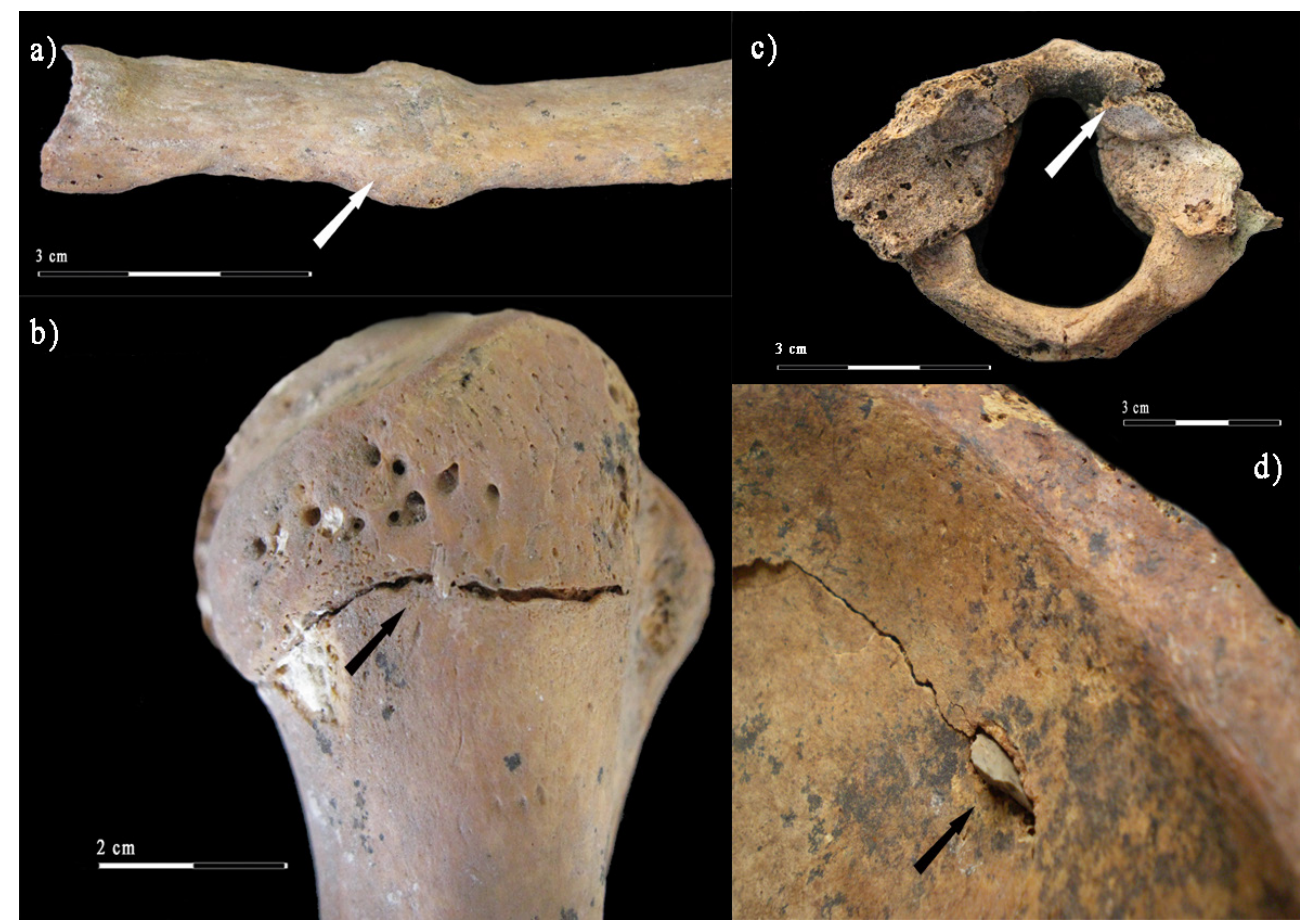

Figura 2. (a) Callo osificado en la costilla de un individuo masculino adulto medio del sitio Orejas de Burro; (b) fractura lineal en el sector latero-posterior del cuello de un húmero derecho perteneciente a un individuo masculino del sitio Orejas de Burro, con evidencia de remodelación ósea (flecha); (c) fractura del arco anterior del atlas de un individuo de sexo indeterminado del sitio Shamakush, con evidencia de remodelación; d) punta de proyectil inserta en el ilion (flecha) de un individuo masculino procedente del norte de Tierra del Fuego, sin evidencia de remodelación ósea. Imágenes tomadas de Suby (2014) y Suby y Guichón (2010).

(a) Ossified callus in the rib of a middle adult male individual from the Orejas de Burro site; (b) linear fracture in the lateral-posterior neck of a right humerus belonging to a male individual from the Orejas de Burro site, with evidence of bone remodeling (arrow); (c) fracture of the anterior arch of the atlas with evidence of bone remodeling in an individual of undetermined sex from the Shamakush site; $(d)$ projectile point inserted in the ilium (arrow), without evidence of bone remodeling in a male individual from the North of Tierra del Fuego. Images taken from Suby (2014) and Suby and Guichón (2011). 
$26,6 \%$ (4/15) de los individuos, mientras que en el 26,8\% (4/15) restante no se especificó la causalidad.

A nivel geográfico, la subregión con mayor frecuencia de traumas fue el norte de Tierra del Fuego $(18,2 \% ; 6 / 33)$, seguido por Santa Cruz/Magallanes $(17,1 \% ; 7 / 41)$ y en menor medida por el sur de Tierra del Fuego $(3,8 \%$; 2/52) (Tabla 3). Aunque los reportes resultaron más frecuentes en las regiones del norte con respecto al sur de Tierra del Fuego, en ningún caso las diferencias fueron estadísticamente significativas. Se observó una mayor tendencia de individuos masculinos que femeninos con traumas en Santa Cruz/Magallanes y el norte de Tierra del Fuego, mientras que en el sur de Tierra del Fuego los únicos dos reportes de traumas fueron identificados en restos de individuos indeterminados (Tabla 3). Si se tienen en cuenta los eventos asignados a violencia interpersonal, la mayor frecuencia de individuos afectados proceden del norte de Tierra del Fuego $(15,1 \% ; 5 / 33)$, seguido por los restos de Santa Cruz/Magallanes $(4,9 \% ; 2 / 41)$, aunque esta diferencia no resulta estadísticamente significativa $(\mathrm{p}=0,32)$. Por el contrario, ningún signo de violencia fue reportado hasta el momento en restos del sur de Tierra del Fuego. En este caso, solo la diferencia entre las subregiones norte de Tierra del Fuego y sur de Tierra del Fuego resultó estadísticamente significativa $(\mathrm{p}=0,01)$.

Respecto de la cantidad de individuos afectados, las lesiones traumáticas se registraron principalmente en la subregión de Santa Cruz/Magallanes (46,7\%; 7/15), seguido por el norte de Tierra del Fuego $(40 \% ; 6 / 15)$ y en menor medida en el sur de Tierra del Fuego (13,3\%; $2 / 15$ ) (Tabla 2), aunque ninguna de estas diferencias resultaron significativas. Por su parte, el mayor porcentaje de lesiones se documentó en el norte de Tierra del Fuego $(53,57 \% ; 15 / 28)$, seguido por Santa Cruz/Magallanes $(39,3 \% ; 11 / 28)$ y sur de Tierra del Fuego $(7,13 \% ; 2 / 28)$. Las diferencias fueron estadísticamente significativas entre norte y sur de Tierra del Fuego y Santa Cruz/ Magallanes y sur de Tierra del Fuego $(p<0,001)$.

Desde una perspectiva temporal, los traumas en Patagonia Austral abarcan todo el periodo comprendido entre los ca. 3.500 y 300 años AP. Durante el lapso 3.5001.700 años AP se registró un 28,6\% de individuos con traumas (4/14), mientras que durante el rango 1.100300 años AP se observó el 21,7\% (5/23) ( $\mathrm{p}=0,7)$. Este resultado se encuentra condicionado por la escasez de esqueletos para momentos más tempranos, y de fechados radiocarbónicos en todos los individuos con traumas.

\section{Discusión}

\section{Interpretación de lesiones traumáticas en Patagonia Austral}

Los reportes de traumas óseos en Patagonia Austral implicaron solo alteraciones muy evidentes, especialmente cuando se tratan de inclusiones de proyectiles en algunas unidades anatómicas. Por el contrario, no fueron mencionados traumas leves que no hayan producido fracturas considerables. Esto podría deberse a la ausencia de análisis más exhaustivos, con objetivos específicos de búsqueda de este tipo de evidencia. Sin embargo, en el estado actual de las investigaciones realizadas es interesante señalar algunas tendencias.

Los elementos óseos más afectados por traumas en Patagonia Austral son las costillas, cráneos y huesos largos. Estas unidades anatómicas son las que presentan los porcentajes más elevados de lesiones traumáticas en restos humanos, independientemente de las características de la muestra y el contexto de estudio, debido a que se localizan en sectores del esqueleto más expuestos a los golpes (Brickey 2006; Judd 2008; Jurmain 1991; Jurmain y Bellifemine 1997, entre otros). En cambio, los traumas son menos recurrentes en vértebras y huesos cortos, generalmente producidos por situaciones de estrés y fuerzas compresivas en sentido cefalocaudal (p.ej., sitio Puesto Pescador 1; Suby et al. 2008). Por lo tanto, esta distribución anatómica de las lesiones es similar a la registrada en otras muestras arqueológicas (Lovell 1997; Merbs 1989).

De acuerdoconel tiempode curación de los traumas, la mayoría de las lesiones identificadas ocurrieron antes de la muerte del individuo, especialmente cuando se tratan de fracturas de huesos largos, vértebras y costillas, lo que indica que los eventos traumáticos no fueron letales, aunque algunos casos pudieron comprometer ciertos aspectos de la salud y condición física, al menos temporalmente (p.ej., Brickley 2006; Lovell 1997). En el caso de las fracturas costales, aunque los autores no mencionan consecuencias en la salud de los individuos, varios estudios han demostrado que lesiones de este tipo podrían producir limitaciones respiratorias, o incluso daños en el bazo o el hígado, en especial en aquellos individuos que tenían tres o más fracturas costales (Brickley 2006; Sirmati et al. 2003). También la fractura femoral identificada en los restos del sitio Palermo Aike (Suby 2014), que produjo el acortamiento de la extremidad inferior, pudo haber causado el desarrollo de un defecto físico y provocado dificultades en ciertas actividades asociadas a la locomoción.

De acuerdo con las interpretaciones realizadas por diferentes autores, las lesiones antemortem fueron causadas por accidentes o por episodios de violencia interpersonal (Constantinescu 2003; L'Heureux y Amorosi 2009; Underdown 2012). En cambio, todas las lesiones de tipo perimortem fueron relacionadas a heridas por proyectiles líticos en la pelvis y golpes en el cráneo que pudieron ser intencionales, probablemente a través del uso de bolas de boleadoras (Suby y Guichón 2010; Suby et al. 2008), lo que indica claros eventos de violencia interpersonal. Sobre este aspecto, resultará 
interesante llevar a cabo análisis experimentales (Otero 2018), teniendo en cuenta las dimensiones y características de los artefactos descritos para la región, que pudieron haber sido utilizados para provocar lesiones traumáticas.

Aunque no se detectaron diferencias estadísticamente significativas, es destacable que todas las lesiones provocadas por violencia interpersonal ocurrieran en los grupos que ocuparon las subregiones de Santa Cruz/ Magallanes y norte de Tierra del Fuego, afectando en un porcentaje elevado a individuos masculinos, al igual que la mayoría de los casos de traumas en general. Además, las subregiones de Santa Cruz/Magallanes y norte de Tierra del Fuego mostraron diferencias significativas en el total de traumas respecto del sur de Tierra del Fuego, algunos de los cuales no es posible descartar que hayan sido producidos por hechos violentos. Esto podría dar cuenta de circunstancias de tensión social tanto a nivel intra como intergrupal en pequeñas bandas de cazadoresrecolectores con estilos de vida pedestre y altamente móviles. Este escenario contrasta con los grupos que habitaron las porciones más australes de Patagonia, caracterizado por un modo de vida cazador-recolector marítimo, donde por el momento no se han detectado individuos con evidencias de lesiones violentas. Sin embargo, según la compilación etnográfica realizada por Prieto y Cárdenas (2007) relacionada con la violencia en las poblaciones Fuego-Patagónicas, estas sociedades practicaban con frecuencia la violencia ya sea a nivel marital, interétnica e intraétnica. En este último caso, era común la guerra a través de pequeños ataques sorpresivos y emboscadas, dañando principalmente a individuos adultos de ambos sexos (Gusinde 1986 [1937]; Prieto y Cárdenas 2007; ver una síntesis en Gat 1999). La ausencia de reportes en el sur de Tierra del Fuego podría deberse a la escasez de estudios sistemáticos específicos orientados a la búsqueda de evidencias traumáticas, por lo que serán necesarios nuevos estudios particularmente en restos de esta subregión.

La mayoría de los individuos con traumas carecen de información temporal (Tabla 2). Cuando se analizan aquellos que pueden ser contextualizados cronológicamente, no se observan diferencias entre los traumas producidos antes y después de los ca. 1.100 años AP, momento aproximado para el cual se propone un aumento en la densidad poblacional de Patagonia Austral (Barberena 2008; Pallo y Ozán 2014; Suby et al. 2017) y ciclos intermitentes de cambios climáticos (p.ej., Anomalía Climática Medieval (ACM); Borromei et al. 2007; Haberzetll et al. 2005; Stine 2000) que pudieron, en algunos casos, haber impactado en la organización conductual de los grupos humanos, en la competitividad por el uso de territorios y recursos, en las relaciones sociales y un aumento de la tensión social y los conflictos. Los resultados detallados en este trabajo no reflejan un aumento en las tensiones sociales a partir del estudio de traumas, que acompañen los cambios organizacionales propuestos para la región, como fue sugerido en trabajos previos en escalas de sitio (p.ej., L’Heureux y Amorosi 2009). Los sesgos en las muestras son siempre una posible explicación, en este caso debido al mayor número de individuos que corresponden a los últimos 2.000 años AP. Sin embargo, no es posible descartar que aun en periodos de aumento demográfico, los cambios sociales no implicaran necesariamente un aumento de los episodios violentos en la región. La muestra analizada igualmente limita aportar datos acerca de posibles diferencias en las frecuencias de traumas antes del Holoceno Tardío, como producto del reducido número de individuos asignados a momentos previos a los ca. 3.500 años AP.

Para el periodo post-contacto no se registraron lesiones violentas, aunque son pocos los individuos con fechados asignables a este momento (Suby et al. 2017). Durante el contacto se introdujo el uso de armas blancas, así como el vidrio como materia prima para la confección de proyectiles en la región continental de Patagonia Austral (Pallo y Borrazo 2017). Por lo tanto, futuras búsquedas de episodios de violencia producidos durante este periodo deberán considerar este tipo de evidencias.

\section{Una aproximación comparativa de los traumas en Patagonia}

El estudio de traumas y fracturas en los esqueletos humanos de grupos cazadores-recolectores de Patagonia, considerando como su límite norte al río Colorado, presenta importantes diferencias en cuanto a la sistematicidad, escalas espaciales y temporales, la composición de las muestras y las preguntas y los problemas arqueológicos afrontados, entre otros factores. Como ocurre en Patagonia Austral, varias investigaciones bioarqueológicas han abordado el estudio de traumas como parte del análisis osteobiográfico de los esqueletos. En pocas ocasiones se han realizado investigaciones con problemas enfocados en evaluar escenarios de violencia interpersonal o accidentes generados en la vida cotidiana, ni se han integrado e interpretado los datos a una discusión en el marco de procesos y dinámicas sociales.

Los análisis más sistemáticos de trauma y con preguntas e hipótesis arqueológicas fueron realizados en el Noreste de Patagonia. Los estudios realizados por Gordón $(2011,2014,2015)$ y Barrientos y Gordón (2004) han sido los más relevantes para discutir aspectos sociales relacionados con la violencia interpersonal. Gordón (2011) analizó 797 cráneos alojados en diversas instituciones pertenecientes a individuos subadultos y adultos, de ambos sexos, procedentes de los cursos 
inferiores de los ríos Colorado, Negro y Chubut, correspondientes a los últimos 4.000 años AP. La autora dividió la muestra a nivel temporal (Holoceno Medio, Tardío inicial, Tardío final y Post-contacto) y a nivel espacial (grupo norte y sur). El grupo norte se conformó por cráneos provenientes de las áreas circundantes a los ríos Colorado y Negro $(\mathrm{n}=436)$ y el grupo sur por individuos de la zona del Río Chubut ( $\mathrm{n}=361$ ). En ambos grupos se registraron perforaciones con y sin inclusión, depresiones, marcas de corte y fracturas lineales (Gordón 2011). Los resultados indicaron la presencia de un $17,56 \%$ de lesiones traumáticas, preferentemente localizadas en el grupo norte $(21,11 \%)$ en relación con el grupo sur $(13,29 \%)$. En el grupo norte los individuos masculinos presentaron mayor porcentaje de traumas respecto a los femeninos, en cambio en el grupo sur no se observaron diferencias en términos estadísticos entre los sexos (Gordón 2014). Respecto de la edad, en ambos conjuntos los adultos presentaron un mayor porcentaje de traumas que los subadultos. Además, no se observaron diferencias estadísticamente significativas en los porcentajes de lesiones a través del tiempo, excepto hacia momentos históricos donde aumenta la frecuencia de traumas, especialmente relacionado con el uso de armas blancas. Según Gordón (2014) los grupos más septentrionales presentan patrones de violencia correspondientes a un estado de conflicto frecuente, involucrando preferentemente a masculinos adultos, tanto en tiempos prehispánicos como posthispánicos. En cambio, el grupo del sur mostró un patrón diferente, que podría relacionarse a contextos de violencia doméstica, ataques o redadas sorpresivas, dañando a ambos sexos por igual. Según la autora, el predominio de violencia no letal entre las mujeres apoya esta idea (Gordón 2011).

Por su parte, Flensborg $(2011,2012,2015)$ realizó estudios específicamente en el curso inferior del Río Colorado, y reportó $26,86 \%$ de traumas y fracturas durante el Holoceno Tardío (ca. 3.000-250 años AP) sobre una muestra compuesta por 67 individuos adultos y adolescentes de ambos sexos. Las lesiones se localizaron principalmente en los cráneos y se trata en su mayoría de fracturas lineales y depresiones de diferentes dimensiones y formas. En el postcráneo se registraron fracturas en huesos largos, cortos y en la cintura escapular e inclusiones de puntas de proyectil en cuerpo vertebral y escápula (Flensborg 2012). La mayoría de las lesiones presentaban remodelación ósea, indicando la supervivencia de los individuos. Además, los traumas habrían sido causados por eventos accidentales y en menor medida por violencia interpersonal. No se registraron cambios en los porcentajes de lesiones traumáticas a través del tiempo, aunque hacia los últimos ca. 1.000 años AP se observó una mayor diversidad de tipos de traumas y las únicas evidencias claras de violencia interpersonal (p.ej., puntas de proyectil incrustadas en el tejido óseo). Los resultados coinciden en líneas generales con las tendencias reportadas por Gordón (2011), ya que no se observó un aumento significativo de la violencia y el conflicto entre los grupos cazadores-recolectores del Noreste de Patagonia durante los últimos ca. 1.000 años AP, aún en el marco de un proceso de complejización social, demarcación territorial, aumento demográfico y mayor tensión social (Barrientos y Gordón 2004; Martínez et al. 2017).

Por el contrario, en la costa norte del Golfo San Matías, si bien no se realizó un análisis exhaustivo de los esqueletos humanos correspondientes al Holoceno Tardío (ca. 3.100-400 años AP), se observaron algunos casos de traumas óseos. En este sentido, se reportaron lesiones de tipo miositis osificante en cráneo y húmero; trauma en calcáneo e impacto de punta de proyectil en un cráneo. En todos los casos corresponden a individuos masculinos, tanto adultos jóvenes como medios (García Guraieb et al. 2010; Mariano 2009). Teniendo en cuenta todos los individuos provenientes del área (NMI=54), el porcentaje de traumas es de $3,71 \%$.

En el valle medio del Río Negro fueron registradas escasas lesiones traumáticas en individuos del Holoceno Tardío (ca. 3.000-900 años AP). Se documentaron fracturas incompletas remodeladas en el cuerpo de tres costillas de un individuo masculino adulto medio y una fractura completa remodelada en el tercio proximal de la diáfisis del fémur derecho de un individuo femenino adulto medio (Prates et al. 2010). Ambos casos habrían sido generados por eventos accidentales. Teniendo en cuenta el total de individuos recuperados en esta área, $(\mathrm{NMI}=29)$, el porcentaje de traumas es de 6,89\%.

Las investigaciones arqueológicas realizadas en el norte de la provincia de Chubut y el valle inferior del Río Chubut, han dado cuenta de la presencia de 137 individuos de ambos sexos y diferentes categorías de edad, para una cronología entre los ca. 7.400-200 años AP. Si bien hasta el momento no se han realizado análisis sistemáticos para estudiar traumas, Gómez Otero (2012) menciona que algunos de los esqueletos rescatados tenían puntas de proyectil clavadas en los huesos o lesiones en el cráneo, lo que sugiere situaciones de enfrentamiento intergrupal, tal vez relacionadas con el uso o control del territorio o de determinados recursos básicos. Ejemplos de esto son tres individuos recuperados del sitio El Golfito (un masculino adulto joven y dos adultos de sexo indeterminado) con evidencia de impacto de proyectiles en la columna vertebral. En el sitio Rawson (ca. 440 años AP) se halló un individuo femenino y un masculino, adultos jóvenes, con puntas de proyectil en costillas, pelvis, escápula y cráneo (Gómez Otero y Dahinten 1997-1998).

En el Lago Salitroso (Noroeste de Santa Cruz) se realizaron estudios paleopatológicos sistemáticos en 
toda la muestra recuperada de la cuenca homónima pertenecientes al Holoceno Tardío (ca. 3.000-300 años AP; García Guraieb 2010). Con respecto a los procesos traumáticos, se observaron bajas frecuencias a nivel de individuos $(10,2 \% ; 5 / 49)$ y consisten en fracturas en el hueso frontal, nasal, hemimaxilar del cráneo, fracturas en vértebras, clavículas e inclusiones de puntas de proyectil en la columna vertebral. Estos traumas registrados en adultos de ambos sexos fueron interpretados como parte de eventos accidentales, avulsiones y violencia interpersonal.

En Patagonia Austral se observó una baja frecuencia de lesiones traumáticas que se asemeja a los porcentajes reportados para otras regiones de Patagonia, en particular a los registrados en el Lago Salitroso (Tabla 4). Por el contrario, se diferencia de la porción más septentrional de Patagonia, donde las prevalencias de traumas registradas son mayores. Particularmente, el curso inferior del Río Colorado muestra porcentajes notablemente mayores a los registrados en otras regiones de Patagonia, aunque se deben considerar aspectos particulares del conjunto óseo, caracterizados por entierros secundarios múltiples, lo que podrían involucrar sesgos en la estimación de la prevalencia de traumas a nivel de individuos (Flensborg 2012). Sin embargo, cuando se tienen en cuenta escalas espaciales más acotadas en Patagonia Austral se observa que el norte de Tierra del Fuego y Santa Cruz/ Magallanes presentan porcentajes de traumas (18,2\% y $17,1 \%$, respectivamente) similares al Noreste de Patagonia y semejanzas en los tipos de lesiones (p.ej. depresiones craneanas, fracturas costales, inclusiones de puntas de proyectil), por lo que es posible que los grupos cazadores-recolectores que ocuparon la región de Patagonia en general estuvieran expuestos a similares circunstancias accidentales (p.ej., caídas) que ocasionaron traumas óseos, y a escasas situaciones de violencia física. Si bien fueron registrados traumas en ambos sexos, los individuos masculinos presentan en general las frecuencias más elevadas respecto de los femeninos, por lo que podría haber una exposición diferencial a los traumas entre ambos sexos. Esta tendencia coincide con la mayoría de los estudios arqueológicos y etnográficos en cazadores-recolectores (p.ej., Gat 1999; Gordón 2011; Walker 2001).

\section{Conclusiones}

Los estudios de traumas en Patagonia Austral se han limitado casi exclusivamente a la descripción de estudios de caso. Este trabajo propone un primer análisis de síntesis de los reportes más recientes realizados sobre restos humanos, detallando las características de las lesiones y sus interpretaciones. Los resultados preliminares muestran en general una baja prevalencia
Tabla 4. Frecuencia de traumas por individuo en distintas regiones de Patagonia. Nota: $\mathrm{NE}=$ noreste $\mathrm{CIRC}=$ curso inferior del río Colorado; CNGSM= costa norte del Golfo San Matías; $\mathrm{CMRN}=$ curso medio de Río Negro; $\mathrm{CCSP}=$ costa centro septentrional de Patagonia.

Frequency of traumas per individual in different regions of Patagonia. Note: $N E=$ northeastern; $C I R C=$ lower course of Colorado River; $C N G S M=$ north coast of San Matías

Gulf; $C M R N=$ middle course of Negro River; $C C S P=$ northern central coast of Patagonia.

\begin{tabular}{lcc}
\hline \multicolumn{1}{c}{ Región } & N & $\%$ \\
\hline NE Patagonia & 797 & 17,56 \\
CIRC & 67 & 26,86 \\
CNGSM & 54 & 3,71 \\
CMRN & 29 & 6,89 \\
CCSP & 137 & 3,64 \\
Lago Salitroso & 49 & 10,2 \\
Patagonia Austral & 119 & 12,6 \\
\hline
\end{tabular}

de evidencias traumáticas en los restos humanos de Patagonia Austral, en mayor frecuencia en individuos masculinos y más concentrados en las subregiones de Santa Cruz/Magallanes y norte del Tierra del Fuego que en el sur de Tierra del Fuego, en especial para los asignados a episodios de violencia interpersonal. En este caso, los resultados indican que los grupos humanos no estuvieron exentos de los enfrentamientos y conflictos sociales, pero muy probablemente los comportamientos violentos de tipo letales no habrían sido una estrategia recurrentemente utilizada.

Los porcentajes generales de traumas se asemejan a otras áreas de Patagonia Meridional, y se diferencian del norte de Patagonia. Sin embargo, cuando se tiene en cuenta la información segregada por subregiones de Patagonia Austral, las prevalencias en Santa Cruz/ Magallanes y norte del Tierra del Fuego son similares a las registradas en el Noreste de Patagonia. Por el momento resulta prematuro asignar causas particulares a la mayoría de los traumas, y los potenciales escenarios socio-demográficos en los cuales pudieron ocurrir los mismos. Aunque existió un mayor aumento demográfico hacia el último milenio, parece no haber contribuido a una mayor competencia y acceso a recursos y enfrentamientos entre grupos, en base a los datos publicados hasta el momento. Evaluaciones más extensas y detalladas permitirán ajustar esta hipótesis.

Los nuevos estudios deberán contemplar análisis detallados de las características de los traumas y discutir las fuerzas y mecanismos involucrados. Temas relacionados con los mecanismos de la violencia, los motivos disparadores de este tipo de comportamiento y el rol de la violencia sobre las mujeres, infantes y niños bajo perspectivas bioculturales actuales del tratamiento de los traumas (Allen y Jones 2014; Martin et al. 2012; Turner y Klaus 2016) aportarán nuevos datos para 
comprender fenómenos sociales relacionados con la organización y adaptación de los grupos humanos a la región. Proyectos de investigación en marcha que involucran el estudio de muestras de Patagonia Austral podrán ofrecer mejores estimaciones e inferencias más ajustadas sobre la prevalencia de los traumas y sus significados culturales en las poblaciones humanas que vivieron allí durante el Holoceno.
Agradecimientos: Este trabajo fue financiado por el Proyecto PICT 0191-2016. Agradecemos a los Dres. Lorena L’Heureux y Leandro Luna por los valiosos comentarios realizados a una versión previa del manuscrito que mejoraron el trabajo. A tres evaluadores anónimos, quienes ayudaron a mejorar este trabajo. Todo lo expuesto aquí es responsabilidad de los autores.

\section{Referencias Citadas}

Adams, B.J. 2007. Forensic Anthropology. Infobase Publishing. New York.

Alfonso-Durruty, M.P, E. Calás y F. Morello 2011. Análisis bioantropológico de un enterratorio humano del Holoceno tardío en Cabo Nose, Tierra del Fuego, Chile. Magallania 39 (1):147-162.

Allen, M.W. 2014. Hunter-gatherer violence and warfare in Australia. En Violence and Warfare among Hunter-Gatherers, editado por M.W. Allen y T.L. Jones, pp. 97-111. Left Coast Press, Inc., New York.

Allen, M.W. y T.L. Jones (eds.) 2014. Violence and Warfare among Hunter-Gatherers. Left Coast Press, Inc., New York.

Aufderheide, A. y C. Rodríguez Martin 1998. The Cambridge Encyclopedia of Human Paleopathology. Cambridge University Press, Cambridge.

Barberena, R. 2008. Arqueología y Biogeografía Humana en Patagonia Meridional. Sociedad Argentina de Arqueología, Buenos Aires.

Barrientos, G. y F. Gordón 2004. Explorando la relación entre nucleamiento poblacional y violencia interpersonal durante el Holoceno tardío en el noreste de Patagonia (República Argentina). Magallania 32 (1):53-69.

Bennike, P. 2008. Trauma. En Advances in Human Paleopathology, editado por R. Pinhasi y S. Mays, pp. 329-362. John Wiley \& Sons, Chichester.

Berón, M.A. 2012. Patrones de violencia en sociedades preestatales: tipificación de eventos a partir de diferentes casos entre cazadoresrecolectores de la Pampa occidental argentina. Una propuesta. En Indicadores Arqueológicos de Violencia, Guerra y Conflicto en Sudamérica, editado por J. López Mazz y M.A. Berón, pp. 81110. Departamento de Publicaciones, Unidad de Comunicación de la Universidad de la República (UCUR), Montevideo.

Berryman, H.E. y S.A. Symes 1998. Recognizing gunshot and blunt cranial trauma through fracture interpretation. En Forensic Osteology: Advances in the Identification of Human Remains, editado por K.J. Reichs, pp. 333-352. Charles C. Thomas, Springfield.

Borrero, L.A. y R. Barberena 2006. Hunter-gatherer home ranges and marine resources. An archaeological case from Southern Patagonia. Current Anthropology 47 (5):855-867.

Borromei, A.M., A. Coronato, M. Quattrocchio, J. Rabassa, S. Grill y C. Roig 2007. Late Pleistocene - Holocene environments in Valle Carbajal, Tierra del Fuego, Argentina. Journal of South American Earth Sciences 23 (4):321-335.

Brickley, M. 2006. Rib Fractures in the archaeological record: A useful source of sociocultural information? International Journal of Osteoarchaeology 16:61-75.
Buikstra, J.E. y D.H. Ubelaker 1994. Standards for data collection from human skeletal remains. Arkansas Archaeological Survey Research Series $\mathrm{N}^{\circ} 44$, Arkansas.

Constantinescu, F. 2003. Obsidiana verde incrustada en un cráneo Aoinikenk: ¿tensión social intraétnica... o interétnica? We'll never know!. Magallania 31:149-153.

Flensborg, G. 2011. Lesiones traumáticas en cráneos del sitio Paso Alsina 1. Explorando indicadores de violencia interpersonal en la transición pampeano-patagónica oriental (Argentina). Intersecciones en Antropología 12:155-166.

Flensborg, G. 2012. Análisis Paleopatológico en el Curso Inferior del Río Colorado (Pcia. de Buenos Aires). Exploración y Evaluación del Estado de Salud de Sociedades Cazadoras-Recolectoras en el Holoceno Tardío. Tesis Doctoral inédita. Facultad de Ciencias Sociales, Universidad Nacional del Centro de la Provincia de Buenos Aires, Olavarría.

Flensborg, G. 2015. Health and disease of hunter-gatherer groups from the eastern Pampa-Patagonia transition (Argentina) during the Late Holocene. Anthropological Science 124 (1):29-44.

Galloway, A. (ed.) 1999. Broken Bones: Anthropological Analysis of Blunt Force Trauma. CC Thomas, Nueva York.

García Guraieb, S., 2010. Bioarqueología de Cazadores-Recolectores del Holoceno Tardío de la Cuenca del Lago Salitroso (Santa Cruz): Aspectos Paleodemográficos y Paleopatológicos. Tesis Doctoral inédita. Facultad de Filosofía y Letras, Universidad de Buenos Aires, Buenos Aires.

García Guraieb, S., C. Mariano y C. Favier Dubois 2010. El Buque Sur: un entierro primario múltiple de 2300 años en la costa del Golfo San Matías, Río Negro, Argentina. Magallania 38 (1):137-148.

Gat, A. 1999. The pattern of fighting in simple, small-scale, prestate societies. Journal of Anthropological Research 55:563583.

Gómez Otero, J. y S. Dahinten 1997-98. Costumbres funerarias y esqueletos humanos: variabilidad y poblamiento en la costa nordeste de la provincia del Chubut (Patagonia argentina). Relaciones de la Sociedad Argentina de Antropología XXII:101-124.

Gómez Otero, J. 2012. La importancia de rescatar los enterratorios humanos en riesgo: experiencias en el nordeste de la provincia de Chubut. Cazadores Recolectores del Cono Sur 4:15-33.

Gordón, F. 2011. Dinámica Poblacional, Conflicto y Violencia en el Norte de Patagonia durante el Holoceno Tardio: un Estudio Arqueológico. Tesis Doctoral inédita. Facultad de Ciencias Naturales y Museo, Universidad Nacional de La Plata, La Plata. 
Gordón, F. 2014. Conflict and Interpersonal Violence in Holocene Hunter-Gatherer Populations from Southern South America. En Violence and Warfare among Hunter-Gatherers, editado por M.W. Allen y T.L. Jones, pp. 133-148. Left Coast Press, Inc., New York.

Gordón, F. 2015. Bioarchaeological patterns of violence in north Patagonia (Argentina) during the late Holocene. Implications for the study of population dynamics. International Journal of Osteoarchaeology 25 (5):625-636.

Gurdjian, E.S. 1975. Impact Head Injury, Mechanistic, Clinical and Preventive Correlations. Charles $\mathrm{C}$ Thomas, Springfield.

Gusinde, M. 1986 [1937]. Los indios de Tierra del Fuego. Los Yamana. Centro Argentino de Etnología Americana, Buenos Aires.

Haberzetll, T., M. Fey, A. Lucke, N. Maidana, C. Mayr, C. Ohlendorf, F. Schäbitz, G. Schleser, M. Wille y B. Zolitschka 2005. Climatically induced lake level changes during the last two millennia as reflected in sediments of Laguna Potrok Aike, southern Patagonia (Santa Cruz, Argentina). Journal of Paleolimnology 33:283-302.

Jordana, X., I. Galtés, T. Turbat, D. Batsukh, C. García, A. Isidro, P. Giscard y A. Malgosa 2009. The warriors of the steppes: osteologica evidence of warfare and violence from Pazyryk tumuli in the Mongolian Altai. Journal of Archaeological Science 36:1319-1327.

Judd, M.A. 2008. The problem Parry. Journal of Archaeological Science 35:1658-1666.

Judd, M.A. y R. Redfern 2012. Trauma. En A companion to paleopathology, editado por A.L. Grauer, pp. 359-379. Blackwell Publishing Ltd., Chichester.

Jurmain, R.D. 1991. Paleoepidemiology of trauma in a central California population. En Human Paleopathology: Current Synthesis and Future Options, editado por D.J. Ortner y A.C. Aufderheide, pp. 241-248. Smithsonian Institution Press, Washington, DC

Jurmain, R. y V. Bellifemine 1997. Patterns of cranial trauma in a prehistoric population from central California. International Journal of Osteoarchaeology 7:43-50.

Jurmain, R., E.J. Bartelink,A.Leventhal,V. Bellifemine, I. Nechayev, M. Atwood y D. DiGiuseppe 2009. Paleoepidemiological patterns of interpersonal aggression in a prehistoric Central California population from CA-ALA-329. American Journal of Physical Anthropology 139:462-473.

Kozameh, L.F., J.E. Barbosa y H.J. Vidal 2000. Los cazadores de Bahía Valentín, Tierra del Fuego. Su status de salud y enfermedad. En Desde el País de los Gigantes. Perspectivas Arqueológicas en Patagonia, pp. 123-139. Universidad Nacional de la Patagonia Austral, Río Gallegos.

Lessa, A. 2011. Daily risks: A biocultural approach to acute trauma in Pre-colonial Coastal populations from Brazil. International Journal of Osteoarchaeology 21:159-172.

L'Heureux, G. y R. Barberena 2008. Evidencias bioarqueológicas en Patagonia meridional: el sitio Orejas de Burro 1 (Pali Aike, provincia de Santa Cruz). Intersecciones en Antropología 9:11-24.

L'Heureux, G. y T. Amorosi 2009. El entierro 2 del sitio Cañadón Leona 5 (Región de Magallanes, Chile). Viejos huesos, nuevos datos. Magallania 37 (2):41-55.

Lovell, N. 1997. Trauma Analysis in Paleopathology. Yearbook of Physical Anthropology 40:139-70.

Lovell, N. 2008. Analysis and interpretation of skeletal trauma En Biological Anthropology of the Human Skeleton, editado por M.A. Ketzenberg y S.R. Saunders, pp. 341-386. WileyLiss Press, New Jersey.

Mariano, C.I. 2009. Prácticas Mortuorias y Registro Bioarqueológico en la Costa Rionegrina del Golfo San Matías, Argentina. Tesis de Licenciatura inédita. Facultad de Ciencias Sociales. Universidad Nacional del Centro de la Provincia de Buenos Aires, ciudad.

Martin, D.L., R.P. Harrod y V.R. Pérez (eds.) 2012. The Bioarchaeology of Violence. University Press of Florida, Gainesville.

Martin, D.L. y C.P. Anderson 2014. Bioarchaeological and Forensic Perspectives on Violence: How Violent Death is Interpreted from Skeletal Remains. Cambridge University Press, New York.

Martin, D.L. y A.J. Osterholtz 2016. Broken bodies and broken bones: Biocultural approaches to ancient slavery and torture. En New Directions in Biocultural Anthropology, editado por M.K. Zuckerman y D.L. Martin, pp. 471-490. John Wiley \& Sons, Inc., New Jersey.

Martínez, G., F. Santos Valero, G. Flensborg, N. Carden, L. Stoessel, A.P. Alcaraz y E. Borges Vaz 2017. Was there a process of regionalisation in Northeastern Patagonia during the Late Holocene? Journal of Island \& Coastal Archaeology 12:95-114

Mays, S.A. 2010. Human osteoarchaeology in the UK 20012007: A bibliometric perspective. International Journal of Osteoarchaeology 20:192-204.

Merbs, C.F. 1989. Trauma. En Reconstruction of Life from the Skeleton, editado por M.Y. Iscan y K.A.R. Kennedy, pp. 161189. Alan Liss, New York.

Miotti, L., M. Salemme y J. Rabassa 2003. Radiocarbon chronology at Piedra Museo Locality. En Where the South Winds Blow, editado por L. Miotti,, M. Salemme y N. Flegenheimer, pp. 99-104. Centre for the Study of First Americans and Texas A and M University Press, Texas.

Morello, F., L.A. Borrero, M. Massone, C. Stern, A. GarcíaHerbst, R. McCulloch, M. Arroyo-Kalin, E. Calas, J. Torres y A. Prieto 2012. Hunter-gatherers, biogeographic barriers and the development of human settlement in Tierra del Fuego. Antiquity 86:71-87.

Nielsen, A.E. 2007. Armas significantes: tramas culturales, guerra y cambio social en el Sur Andino Prehispánico. Boletín del Museo Chileno de Arte Precolombino 12 (1):9-41.

Orquera, L.A. y E. Piana 2009. Sea nomads of the Beagle Channel in Southernmost South America: Over six thousand years of coastal adaptation and stability. Journal of Island and Coastal Archaeology 4:61-81.

Ortner, D. 2003. Identification of Pathological Conditions in Human Skeletal Remains. Academic Press, San Diego.

Otero, F. 2018. Análisis de Marcas Óseas por Armas Contundentes: una Aproximación Experimental. Tesis de Licenciatura en Criminología y Ciencias Forenses, Universidad Nacional de Río Negro, General Roca.

Pallo, M.C. y I.L. Ozán 2014. Variaciones demográficas y climáticas durante el Holoceno tardío final en Magallania. Comechingonia Virtual, Revista Electrónica de Arqueología VIII (1):20-47.

Pallo, M.C. y K. Borrazo 2017. The archaeology of contact in Southern Patagonia: Some issues to be resolved in the Southwestern Forest. Arts and Humanities Open Access Journal 1 (4):00023. DOI: 10.15406/ahoaj.2017.01.00023. 
Pfeiffer, S. 2016. An exploration of interpersonal violence among Holocene foragers of Southern Africa. International Journal of Paleopathology 13:27-38.

Prates, L., G. Flensborg y P. Bayala 2010. Caracterización de los entierros humanos del sitio Loma de los Muertos (valle medio del río Negro). Magallania 38 (1):147-162.

Prieto, A. 1991. Cazadores tempranos y tardíos en cueva de Lago Sofía 1. Anales del Instituto de la Patagonia 20:75-99.

Prieto, A. y R.A. Cárdenas 2007. The struggle for social life in Fuego-Patagonia. En Latin American Indigenous Warfare and Ritual Violence, editado por R.J. Chacon y R.G. Mendoza, pp. 212233. The University of Arizona Press, Tucson.

Saletta, M.J. 2015. Excavando Fuentes: la Tecnología, Subsistencia, Movilidad y los Sistemas Simbólicos de Shelk'nam, Yámana/Yaghan y Aonikenk entre los Siglos XVI y XX Analizados a Partir de los Registros Escritos y Arqueológicos. Tesis Doctoral inédita. Facultad de Filosofía y Letras, Universidad de Buenos Aires, Buenos Aires.

Santana, J., J. Velasco, J.J. Ibañez y F. Braemer 2012. Crania with mutilated facial skeletons: A new ritual treatment in an Early PrePottery Neolithic B Cranial Cache at Tell Qarassa North (South Syria). American Journal of Physical Anthropology 149:205-2016.

Sauer, N. 1998. The timing of injuries and manner of death: Distinguishing among antemortem, perimortem, and postmortem trauma. En Forensic Osteology: Advances in the Identification of Human Remains, editado por K. Reichs, pp. 321-332. Charles C Thomas, Springfield.

Scott, R.M. y H.R. Buckley 2010. Biocultural interpretations of trauma in two prehistoric Pacific Island populations from Papua New Guinea and the Solomon Islands. American Journal of Physical Anthropology 142 (4):509-518.

Seldes, V. y F.N. Botta 2014. Violence indicators in Quebrada de Humahuaca, Jujuy, Argentina: The Regional Development Period from a regional perspective. Anthropological Review 77 (1):87-109.

Sirmali, M., H. Türüt, S. Topçu, E. Gülhan, U. Yazici, S. Kaya e I. Taștepe 2003. A comprehensive analysis of traumatic rib fractures: morbidity, mortality and management. European Journal of Cardio-Thoracic Surgery 24:133-138.

Stine, S., 2000. On the medieval climatic anomaly. Current Anthropology 41:627-628.

Stodder, A.L.W. 2008. Taphonomy and the Nature of Archaeological Assemblages. En Biological Anthropology of The Human Skeleton, editado por M.A. Katzemberg y S.R. Saunders, pp. 71-114. WileyLiss Inc, New York.

Suby, J.A. 2014. Desarrollos recientes en el estudio de la salud de las poblaciones humanas antiguas de Patagonia Austral. En Avances Recientes en la Bioarqueología Latinoamericana, editado por L.H. Luna, C. Aranda, J.A. Suby. pp. 69-100. Grupo de Investigaciones en Bioarqueología, Buenos Aires.

Suby, J.A. y R.A. Guichón 2010. Los restos óseos humanos de la colección de la misión "La Candelaria" (Rio Grande, Tierra del Fuego). Magallania 38 (2):121-133.

Suby, J.A., R.A. Guichón y A.F. Zangrando 2009. El registro biológico humano de la costa meridional de Santa Cruz. Revista Argentina de Antropología Biológica 11 (1):109-124.

Suby, J., L.H. Luna, C. Aranda y G. Flensborg 2017. First approximation to paleodemography through age-at-death profiles in hunter-gatherers from Southern Patagonia during middle-late Holocene. Quaternary International, http://dx.doi.org/10.1016/j. quaint.2017.04.035.

Suby, J.A., M. Salemme y F. Santiago 2008. Análisis paleopatológico de los restos humanos del sitio Puesto Pescador 1 (Tierra del Fuego). Magallania 36 (1):53-64.

Suby, J.A., A.F. Zangrando y E. Piana 2011. Exploraciones osteológicas de la salud de las poblaciones humanas del Canal Beagle. Relaciones de la Sociedad Argentina de Antropología 36:249-270.

Turner, B.L. y H.D. Klaus 2016. Biocultural perspectives in bioarchaeology. En New Directions in Biocultural Anthropology, editado por M.K. Zuckerman y D. Martin, pp. 429-451. John Wiley \& Sons, Inc. New Jersey.

Underdown, S. 2012. Fitzroy's fighting Fuegians: Comparison of palaeopathological and ethnographic indications of fighting behaviour in an extinct human population. International Journal of Osteoarchaeology 22 (3):373-378.

Villa, P. y E. Mahieu 1991. Breakage patterns of human long bones. Journal of Human Evolution 21:27-48.

Walker, P.L. 2001. A bioarchaeological perspective on the history of violence. Annual Review of Anthropology 30:573-596.

Wedel, V.L. y A. Galloway 2014. Broken Bones. Anthropological Analysis of Blunt Force Trauma. Segunda Edición. Charles S.C. Thomas, Publisher, LTD, Springfield. 
